\title{
Highly Nonconductive Li-Ion Battery Components Successfully Imaged at Very Low Accelerating Voltages
}

\author{
Dipl. Ing. Stefanie Freitag ${ }^{1}$, Dr. Christina Berger ${ }^{1}$, Jeff Gelb ${ }^{1}$, Christian Weisenberger ${ }^{2}$, Dr. Timo
} Bernthaler ${ }^{2}$

1. Carl Zeiss Microscopy GmbH, Munich, Germany

2. Aalen University, Materials Research Institute, Aalen, Germany

Rechargeable Li-ion batteries are meant to be used as reliable power source in portable electronic devices such as cellphones, laptops, cameras, etc. and are becoming increasingly important in the automotive industry and for stationary storage applications. A battery cell comprises an electrochemically coupled positive (anode) and negative (cathode) electrode, electrically isolated from each other by a separator. Only ions can pass the separator, so the direct short circuit of electrons between the electrodes is prevented [1]. Li-ion batteries are sensitive and complex electrochemical energy storage devices incorporating many material types, ranging from conductive metals to very nonconductive polymers, and require a flexible suite of instrumentation for proper characterization. Especially challenging are charging effects and the danger of damage using higher accelerating voltages in the electron microscope. For example the binder which provides the cohesion of the active materials as well as their adhesion to the metallic current collector, is polymeric thus is challenging to image but due to its low conductivity, oversupply needs to be detected and avoided. [2, 3]

In this study, very low voltage imaging was used to investigate anode, cathode, binder and the separator of a Li-ion battery. Voltages of $1 \mathrm{kV}$ down to $100 \mathrm{~V}$ were used to image two commercial prismatic lithium ion battery cells (3.7 V /1500 mAh) which utilized $\mathrm{LiCoO}_{2}$ (LCO) in the positive electrode and graphite in the negative electrode. One cell it it's pristine state and one cell which was subject to an ageing procedure comparable to several years of use in a mobile device were investigated. After discharge the cells were opened in a glove box under protective argon atmosphere. The electrode foils were separated, washed in dimethyl carbonate (DMC), and dried. Small samples were cut from the electrodes and the separator, then placed as obtained on a microscopy stub and secured with a carbon tape as well as molded into an epoxy resin for cross section analysis.

Imaging at low accelerating voltages, revealed additional information beyond the reported structures in batteries. It allowed an increased image fidelity on the extent of particle cracking, the observation of inhomogeneous binder distributions, binder strings and the detection of cubic and spherical shaped objects. Binder strings between particles in the cathode and anode material spanned lengths of $20 \mathrm{~nm}$ up to $3 \mu \mathrm{m}$. The electron beam exposure quickly lead to melting and disappearance of the strings which indicates, that the strings might be polymeric. In addition cubic shaped objects in the cathode were observed, with a few $10 \mathrm{~nm}$ lateral extent and the ones in the separator about $10 \mu \mathrm{m}$ in size. In contrast, in the anode spherical objects ranging from $20 \mathrm{~nm}$ up to $40 \mathrm{~nm}$ in lateral extent were observed. Surprisingly, they did not disappear after cycling, but instead fused to a more closed layer around the anode particles. High magnification images of the sensitive separator revealed that the new one consists of an aligned microstructure within the membrane and the old separator exhibited a more disordered microstructure. This finding might originate from a changed separator material supplier or it is an effect of the ageing, which likely will have an effect on the battery performance e.g. on the impedance. Similar results were found in studies using Raman microscopy [4]. 
It was demonstrated that with the imaging performance especially at very low voltages, highly nonconductive material can be imaged. No further conductive treatment of the sample was needed thus differences of the microstructure before and after ageing of the battery can be observed. These new results underline the importance of high beam stability at lower accelerating voltages, enabling highresolution studies of even the most challenging Li-ion battery material systems.

\section{References:}

[1] Hamann, C.H.; Vielstich, W.: Elektrochemie,4. Auflage, Wiley-VHC Verlag, Weinheim, 2005 [2] Voelker, Paul (2014-04-22). “Trace Degradation Analysis of Lithium-Ion Battery Components”. R\&D. Retrieved April 2015

[3] J. Vetter et al., Journal of Power Sources 147 (2005), 269-28

[4] S. Freitag, C. Berger et al., EN_42_013_184_Integrated-SEM-and-Raman-imaging-of-Lithium-IonBattery.pdf, Application Note, ZEISS Microscopy, Oct. 2015
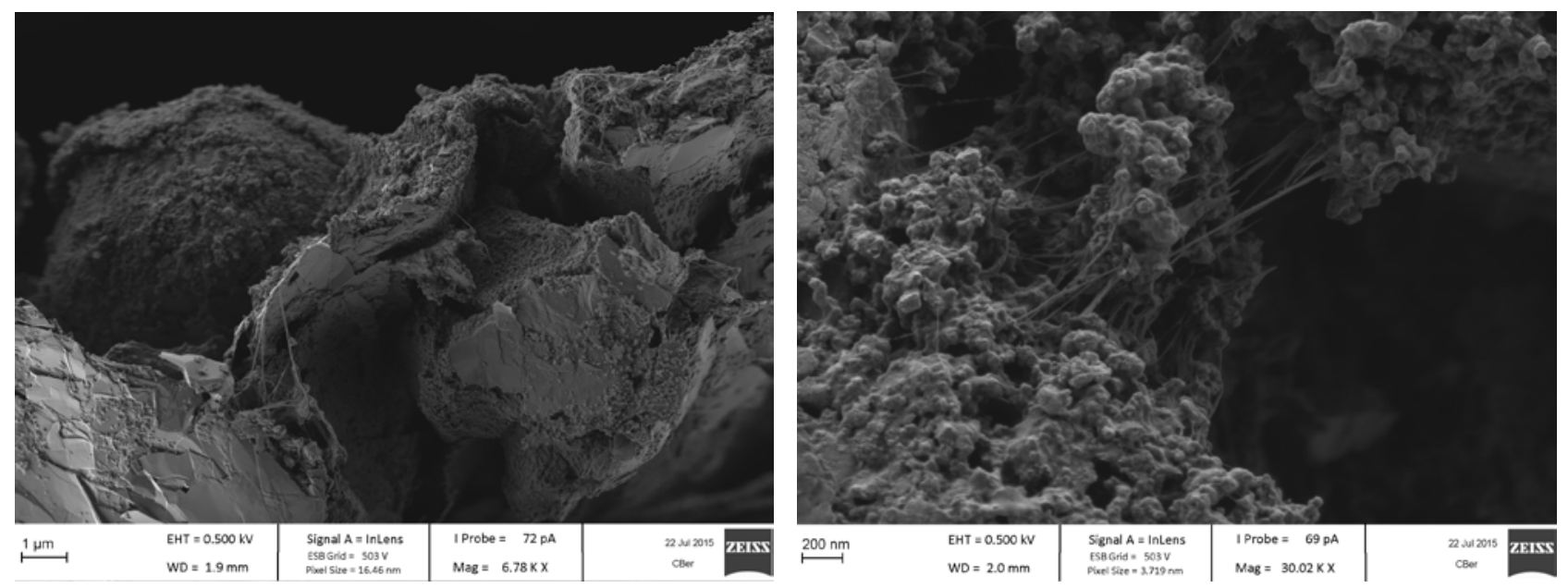

Figure 1. Binder strings imaged at low accelerating voltages, which are spanning over a few $\mu \mathrm{m}$ in the anode material (left) and several hundred nm in the cathode (right)
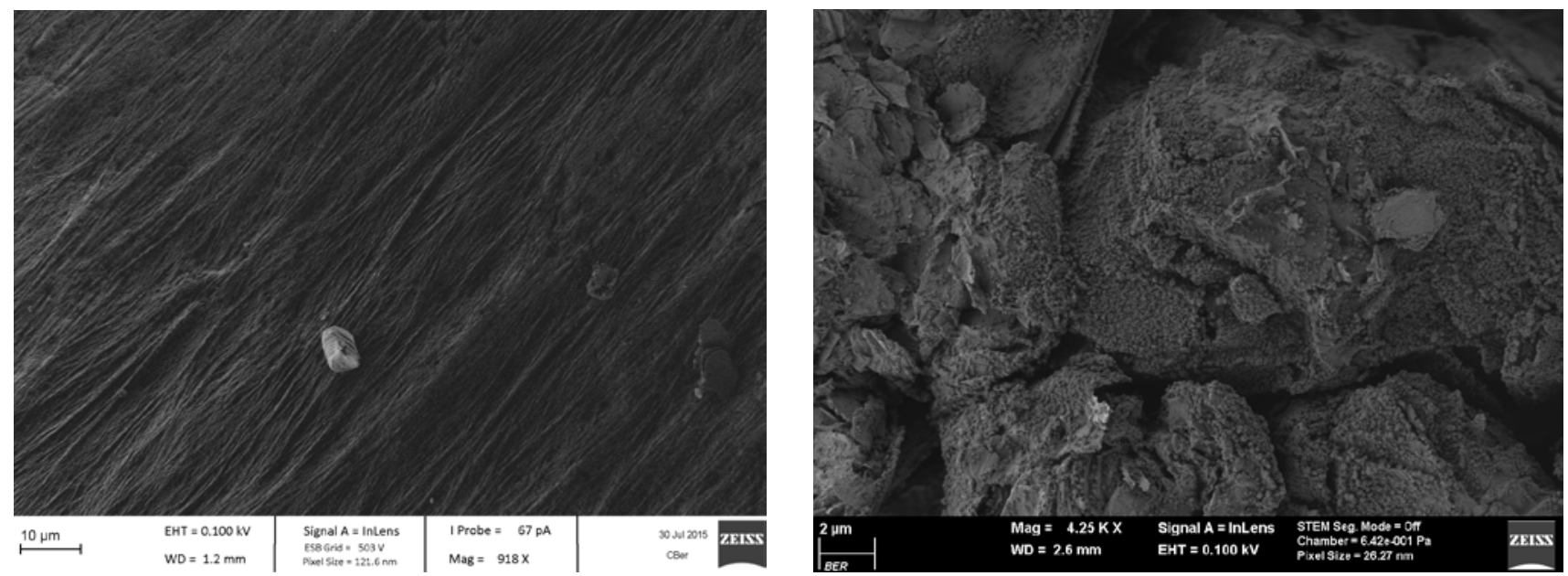

Figure 2. Detected cubic objects in the separator (left) and spherical objects on the surface of anode particles (right) 